\title{
AOR
}

Selected Papers of \#AoIR2019:

The $20^{\text {th }}$ Annual Conference of the Association of Internet Researchers Brisbane, Australia / 2-5 October 2019

\section{ANALYZING PLATFORM POWER: APP STORES AS INFRASTRUCTURAL PLATFORM SERVICES}

\author{
David B. Nieborg \\ University of Toronto \\ José van Dijck \\ Utrecht University \\ Thomas Poell \\ University of Amsterdam
}

February 2019, tech magazine TechCrunch revealed how mobile app developers have been abusing so-called "Enterprise Certificates" (Constine, 2019). Apple grants these special software licenses to businesses to bypass the iOS App Store and distribute apps among employees. What prompted TechCrunch's investigation was earlier reporting on the misuse of these certificates by Facebook and Google. It turned out, smaller app developers also exploited this loophole. TechCrunch pointed to real-money gambling and pornography apps, the latter category being outright forbidden in the App Store.

This story of a heavily regulated app space speaks to the broader question of the evolution and entrenchment of platform power. Apple is one of a very select group of U.S.-based platform operators that shape the flow of public information in the Western world, thereby disrupting societal institutions and industry sectors. As a broad variety of scholars pointed out, there is a severe lack of transparency in how these platforms operate and intervene in public life (Bucher 2018; Gillespie 2018a). This is particularly problematic, as their interventions conflict with vital public values, undermining socioeconomic equality, democratic processes, and the quality of public services (Van Dijck, Poell \& De Waal, 2018). In this paper we examine how platform power is operationalized in the specific case of app stores. We take a first step in developing an analytical framework that critically examines the infrastructural power relations that constitute online platform ecosystems. This involves distinguishing among different levels of platform ecosystem analysis. Building on a relational understanding of power, we propose an analytical vocabulary to systematically interrogate the material power

Suggested Citation (APA): Nieborg, D.B. Van Dijck, J., Poell, T. (2019, October 2-5). Analyzing Platform Power. App Stores as Infrastructural Platform Services. Paper presented at AolR 2019: The $20^{\text {th }}$ Annual Conference of the Association of Internet Researchers. Brisbane, Australia: AolR. Retrieved from http://spir.aoir.org. 
relations among the three main actors active in platform ecosystems: platform operators (e.g. Apple), third party institutions (e.g. app developers, businesses, governments), and end-users (i.e. individuals).

\section{Five levels of platform power}

To better differentiate among the different actors in platform ecosystems, the paper proposes to study platform power at five expanding levels, similar to those of ecological ecosystems. The first level is that of individual actors, who can be end-users or developers, and who typically only yield power in the aggregate. Second, these users constitute populations aggregated by infrastructural platform services. The iOS App Store, we argue, is an example of such a service as it acts as a key access node in Apple's wider platform ecosystem. End-users need access to the App Store to download individual apps of competing platform operators (Facebook and Google) and developers need access to offer their apps and services. Infrastructural platform services operate as markets, connecting different actors. They are the connective glue, so to say, that allows tech companies to govern an opaque and complex ecosystem in which connections are invisible to the public eye and hence largely beyond societal control.

For platform operators there is an incentive to expand its reach beyond individual platform services, which brings us to the third level of platform power: those of company platform ecosystems. For example, Apple has amassed hundreds of millions of users of Apple devices, all of whom are subject to the rules and regulations of governance frameworks set by Apple. Collectively, major platforms companies exercise power on a fourth level: that of the geopolitical platform ecosystem. We distinguish between two dominant ones. U.S.-based platform companies are primarily active in the West, whereas China-based platforms originate and operate in a heavily state-sanctioned environment. Lastly, the highest level of abstraction is the global platform ecosystem, which combines all individual actors and encompasses all levels. This fifth level is likely to become increasingly relevant to scholarship on platform power as platforms have ambitions beyond their respective geopolitical spheres. Studying infrastructural platform services, such as app stores, offers relevant insight into how globally operating platforms are able to set, steer, and bend rules and norms that impact individual actors on the local and national level.

\section{App stores as infrastructural platform services}

The typical unit of analysis in scholarship discussing platform power is either the level of individual actors, company ecosystems, or geopolitical ecosystems. Missing here is the level of infrastructural platform services and how they fit into the higher levels of platform ecosystems. Examples of such services include app stores, but also advertising networks, login services, cloud hosting, payment systems, data analytics, and artificial intelligence (Al) services. We consider these services infrastructural because of their ubiquity and consistent use, which allows for platform power to emerge and to be consolidated (Plantin et al. 2018). App stores, then, offer a standardized, techno-economic architecture for app developers. In the West, to reach billions of mobile media users, app developers have to go through either Apple's iOS App Store or Google's Play Store. Thus, their reach extends far beyond dominant marketplaces in physical domains, such as Walmart or Media Markt. 
In the case of app stores, the paper shows that platform power is not casual or discursive, but highly strategic, uniform, and centralized. First, we examine how endusers and developers are firmly 'locked-in', as it is impossible to transfer data (or apps) outside the proprietary platform walls. Second, the analysis demonstrates that by active gatekeeping, platform operators are able to halt, prohibit, block, or privilege some apps over others. This raises the wider question of market entry and distribution, particular in the case of cultural production (Nieborg \& Poell, 2018). For example, Apple follows a set of subjective and opaque submission guidelines (Gillespie, 2018b), as illustrated by the gambling and pornography apps in our introduction. While notably hard to investigate, our understanding of platform power would benefit from greater insight into this issue, to be gained via developer interviews, reviewing shifts in policy, or surveying apps that are rejected (Hestres, 2013). Third, app stores operators have full control over which apps are featured in their storefronts, deeply impacting individual actors and institutions. Investigating these regimes of platform visibility, we stress, requires a sustained effort that draws on developer interviews, digital methods, and financial analysis.

By interrogating these three instances of platform power at the platform service level, the paper demonstrates that platform power is not a property of one platform itself, but a corollary of a platform's function in the context of other platforms and actors in a dynamic ecosystem. Future research could illuminate how, specifically, other apps stores, or other infrastructural platform services gain, lose, and wield platform power in similar or different ways.

\section{References}

Bucher, T. (2018). If... Then. Algorithmic Power and Politics. Oxford: Oxford University Press.

Constine, J. (2019, February 13). Apple fails to block porn \& gambling 'Enterprise' apps. TechCrunch. Retrieved from https://techcrunch.com/2019/02/12/apple-porn-gamblingapps/.

Gillespie, T. (2018a). Custodians of the Internet. Platforms, Content Moderation, and the Hidden Decisions That Shape Social Media. New Haven: Yale University Press.

Gillespie, T. (2018b). Exodus International. Banned Apps, App Stores, and the Politics of Visibility. In J. W. Morris \& S. Murray (Eds.), Appified. Culture in the age of apps (pp. 51-60). Ann Arbor: University of Michigan Press.

Hestres, L. E. (2013). App neutrality: Apple's App Store and freedom of expression online. International Journal of Communication, 7, 1265-1280.

Nieborg, D. B., \& Poell, T. (2018). The platformization of cultural production: Theorizing the contingent cultural commodity. New Media \& Society, 20(11), 4275-4292. 
Plantin, J. C., Lagoze, C., Edwards, P. N., \& Sandvig, C. (2018). Infrastructure studies meet platform studies in the age of Google and Facebook. New Media \& Society, 20(1), 293-310.

Van Dijck, J., Poell, T., \& De Waal, M. (2018). The Platform Society. Public Values in a Connective World. Oxford: Oxford University Press. 